\title{
Co-administration of Colchicine and Allopurinol alleviates vascular calcification induced by hyperuricemia via suppressing inflammation and oxidative stress
}

\author{
Type
}

Research paper

\section{Keywords}

gout, inflammation, Oxidative stress, colchicine, vascular smooth muscle cells, allopurinol, vascular calcification

\begin{abstract}
Introduction

Oxidative stress and inflammation are involved in the pathogenesis of VC in patients with hyperuricemia, while lowering the uric acid level accordingly reduces the risk of VC. Additionally, both ALL and COL suppress inflammatory response and oxidative stress. However, it is unclear whether novel therapeutic strategies, such as combined administration of COL and ALL, can achieve a better performance in lowering hyperuricemia. Therefore, we aimed to investigate the effect of coadministration of COL and ALL in the treatment of VC.
\end{abstract}

Material and methods

Von Kossa staining was performed to evaluate the aortic vascular calcification in HUC rats treated under different therapeutic conditions. Quantitative real-time PCR was carried out to analyze the expression of genes involved in the pathogenesis of hyperuricemia.

\section{Results}

Combined administration of COL and ALL alleviated the aortic vascular calcification in HUC rats. The aberrant up-regulation of genes related to differentiation, BMP2, RUNX2, OC and ALP, was effectively reversed by the combined treatment of COL and ALL in HUC rats and cellular models. Besides, the dysregulation of enzymes and cytokines involved in oxidative and inflammatory responses was restored by the combined treatment of COL and ALL.

\section{Conclusions}

In this study, we tested the therapeutic effect of ALL combined with COL on the treatment of VC in animals with hyperuricemia by examining their influence on oxidative stress and inflammation. Our work helped to gain a deeper insight into the molecular mechanism of hyperuricemia, and revealed that the efficiency of the combined treatment with COL and ALL out-performed the mono-therapy of any single compound. 
1 Co-administration of Colchicine and Allopurinol alleviates vascular calcification induced

2 by hyperuricemia via suppressing inflammation and oxidative stress

3 Yang $\mathrm{Ji}^{1}$, Maojing Wang ${ }^{1 *}$, Libo Sun ${ }^{2 *}$, Wei Ding ${ }^{3}$, Shanglang $\mathrm{Cai}^{1}$, Qing Zhao ${ }^{1}$

4 1.Department of Cardiology, The Affiliated Hospital of Qingdao University, Qingdao, Shandong

5 Province, China, 266003

6 2.Pharmacy Department, The Affiliated Hospital of Qingdao University, Qingdao, Shandong

7 Province, China, 266003

8 3.Ophthalmology Department, The West Coast New Area People's Hospital of Qingdao,

9 Qingdao, Shandong Province, China, 266400

$10 *$ Corresponding author:

11 Libo Sun

12 Institution: Pharmacy Department, The Affiliated Hospital of Qingdao University, Qingdao,

13 Shandong Province, China, 266003

14 Address: 16 Jiangsu Rd, Qingdao, Shandong Province, China 266005

15 Email: cardiodox @yeah.net

16 and

17 Maojing Wang

Institution: Department of Cardiology, The Affiliated Hospital of Qingdao University, Qingdao,

19 Shandong Province, China, 266003

20 Address: 16 Jiangsu Rd, Qingdao, Shandong Province, China 266003

21 Email: cardioarrythmia@ $163 . c o m$

\section{Abstract}

23 Background: Oxidative stress and inflammation are involved in the pathogenesis of VC in

24 patients with hyperuricemia, while lowering the uric acid level accordingly reduces the risk of VC.

25 Additionally, both ALL and COL suppress inflammatory response and oxidative stress. However, 
it is unclear whether novel therapeutic strategies, such as combined administration of COL and

27 ALL, can achieve a better performance in lowering hyperuricemia. Therefore, we aimed to investigate the effect of co-administration of COL and ALL in the treatment of VC. Methods: Von Kossa staining was performed to evaluate the aortic vascular calcification in HUC rats treated under different therapeutic conditions. Quantitative real-time PCR was carried out to analyze the expression of genes involved in the pathogenesis of hyperuricemia. Results: Combined administration of COL and ALL alleviated the aortic vascular calcification in HUC rats. The aberrant up-regulation of genes related to differentiation, BMP2, RUNX2, OC and ALP, was effectively reversed by the combined treatment of COL and ALL in HUC rats and cellular models. Besides, the dysregulation of enzymes and cytokines involved in oxidative and inflammatory responses was restored by the combined treatment of COL and ALL. Conclusion: In this study, we tested the therapeutic effect of ALL combined with COL on the treatment of VC in animals with hyperuricemia by examining their influence on oxidative stress and inflammation. Our work helped to gain a deeper insight into the molecular mechanism of hyperuricemia, and revealed that the efficiency of the combined treatment with COL and ALL out-performed the mono-therapy of

41 any single compound.

42 Running title: Colchicine and allopurinol affect vascular calcification

43 Key words: GOUT; Allopurinol; Colchicine; Vascular calcification; Vascular smooth muscle 44 cells; Oxidative stress; Inflammation

\section{Abbreviations list:}

$46 \quad$ VC: vascular calcification

47 ALL: allopurinol

48 COL: colchicine;

49 HUC: hyperuricemia;

50 VSMC: vascular smooth muscle cell. 
As an increasingly common chronic disorder of urate crystal deposition, gout specifically affects joints, although it can also affect tissues including ligaments, which is more prevalent in men than women prior to menopause [1]. Medical diagnosis of gout is based upon medical symptoms, such as the presentation of MSU crystals in the SF or peri-articular deposition [2], while the overarching principle of gout management is to reduce serum urate levels to $0.36 \mathrm{mmol} / \mathrm{L}$ or below depending on whether it is tophaceous or non-tophaceous respectively [1]. Meanwhile, hyperuricemia (HUC) refers to an unusual state where the uric acid level in the serum is greater than $7.0 \mathrm{mg} / \mathrm{dL}[3,4]$. HUC is also the basis of gout as well as a hazard factor in various other medical conditions (e.g., severe and persistent kidney conditions, diabetes, as well as metabolic disorders) [5, 6]. Uric acid can also induce the phenotypic changes in kidney tubular cells [7]. HUC induced by chemicals is also related to decreased kidney functions mostly because of improved OS, systemic as well as glomerular high blood pressure, vascular damages, as well as glomerular hypertrophy [8, 9]. Uric acid may also cause OS via other molecular signaling, including systemic inflammation, which plays an essential role in CKD [10]. Zhou and his colleagues discovered that the induction of HUC is accompanied with the entry of macrophages as well as T cells into the kidney, along with the activation of NF-kB, TNF-a, and MCP-1 [11]. Considering that inflammation causes the generation of ROS, uric acid could trigger OS with its pro-inflammatory activity [12]. Similarly, an additional research revealed that the direct exposure of human endothelial cells to uric acid is accompanied with upregulated inflammation as well as the rise in C-reactive protein (CRP) level.

As a first line treatment for urate lowering therapy in the management of gout, allopurinol is swiftly metabolized to oxypurinol, which in turn decreases the level of urate [13, 14]. Regardless of its extensive usage over 40 years, many patients treated by allopurinol still fail to achieve long-term management of gout, thus raising controversial comments about optimal allopurinol dosing in gout patients with chronic kidney disease [14, 15]. Issues regarding unfavorable effects of allopurinol, specifically the allopurinol hypersensitivity, led some medical professionals to restrict the dosing of allopurinol in gout patients with impaired kidney function [15].

Colchicine is an all-natural compound separated from lily plants [16]. As a lipid soluble compound that has been used for more than 1000 years, colchicine is approved by the United States FDA in the therapy of intense gout pain as well as joint inflammation caused by gout [16-19]. Colchicine could block polymerization of tubulin in gout, which prevents the activation of the inflammasome 
[19]. Colchicine has actually been shown to impair cytoskeleton structures as it binds to the heterodimers of tubulin to generate a steady aggregate, leading to the depolymerization of microtubules [20].

85

It has been reported that oxidative stress and inflammation are involved in the pathogenesis of VC in subjects with hyperuricemia, such as those diagnosed with gout, while uric acid lowering treatment accordingly reduces the risk of VC [21-23]. Also, both ALL and COL are reported to suppress the inflammatory response and oxidative stress in acute liver failure or familial mediterranean fever [24, 25]. In this study, by studying the influence of ALL and COL on oxidative stress and inflammation, we aimed to investigate the effect of co-administration of ALL and COL in the treatment of $\mathrm{VC}$ in animals with hyperuricemia.

\section{Materials and Methods}

\section{Animal and treatment}

In this research, $30 \mathrm{SD}$ male rats at an age of 6 weeks old were acquired and placed in a specificpathogen-free (SPF) animal center, which was set to a temperature level of $23{ }^{\circ} \mathrm{C}$ and a humidity level of $60 \%$. The animals were adjusted to the animal center for 7 days, throughout which the animals were provided a regular rodent diet, before they were divided into 5 groups, i.e., 1. SHAM group $(\mathrm{N}=6) ; 2$. HUC group $(\mathrm{N}=6) ; 3$. HUC + COL group $(\mathrm{N}=6) ; 4$. HUC + ALL group $(\mathrm{N}=6)$; and 5. HUC + ALL + COL group $(\mathrm{N}=6)$. To establish the HUC rat model, the rats were provided with a standard chow supplemented with $2 \%$ of potassium oxonate. In the HUC + ALL group, the HUC rats were given an ALL treatment at a dose of $100 \mathrm{mg} / \mathrm{kg}$ by oral intubation after the HUC model was established. In the HUC + COL group, the HUC rats were given a COL treatment at a dose of $1 \mathrm{mg} / \mathrm{kg}$ via i.p. injection after the HUC model was established. In the HUC + ALL + COL group, the HUC rats were administrated of $100 \mathrm{mg} / \mathrm{kg}$ of ALL by oral intubation and $1 \mathrm{mg} / \mathrm{kg}$ of COL by i.p. injection after the HUC model was established. Throughout the entire experiment, the rats were given unrestricted drinking water access. From the day when the HUC model was established, the levels of UA as well as creatinine in the serum were assayed daily by utilizing blood specimens collected under fasting. In the 16th week after the HUC model was established, all rats were sacrificed to harvest their femurs for succeeding evaluations. In addition, Von Kossa staining was done to examine aortic vascular calcification in HUC rats treated under various conditions. Institutional ethical committee has approved the protocol of this study. 
113

114

115

116

117

118

119

120

121

122

123

124

125

126

127

128

129

130

131

132

133

134

135

136

137

138

139

140

In this study, the expression of BMP2, RUNX2, OC, ALP, and TLR2 mRNA was measured using real time PCR. In brief, RNA was separated from collected tissue and cell samples by utilizing a Trizol agent (Invitrogen, Carlsbad, CA) in accordance to the procedure provided by the kit supplier. In the next step, extracted RNA was diluted in water containing RNase-free DNase (Promega, Madison, WI) while the quality of extracted RNA was inspected by gel electrophoresis. Then, the extracted RNA was converted to cDNA by utilizing a PrimeScript RT reagent kit (Takara, Otsu, Shiga, Japan) in accordance to the procedure provided by the kit supplier. In the next step, roughly 50 ng of RNA in each specimen was loaded onto a StepOnePlus Real Time PCR machine (Applied Biosystems, Foster City, CA) to carry out real time PCR using a SYBR Premix Ex Taq II assay kit (Takara, Otsu, Shiga, Japan) in accordance to the procedure provided by the kit supplier. Finally, the relative expression of BMP2 mRNA (Forward Primer: 5' TGTATCGCAGGCACTCAGGTCA-3'; Reverse 5rimer: CCACTCGTTTCTGGTAGTTCTTC-3';), RUNX2 mRNA (Forward Primer: 5'CCCAGTATGAGAGTAGGTGTCC-3’; Reverse Primer:

5 'GGGTAAGACTGGTCATAGGACC-3'), OC mRNA (Forward Primer: 5'CGCTACCTGTATCAATGGCTGG-3';

Reverse

Primer: 5 'CTCCTGAAAGCCGATGTGGTCA-3'), ALP mRNA (Forward Primer: 5'GCTGTAAGGACATCGCCTACCA-3'; Reverse Primer: CCTGGCTTTCTCGTCACTCTCA-3'), and TLR2 mRNA (Forward Primer: 5' CTTCACTCAGGAGCAGCAAGCA-3'; Reverse Primer: 5'ACACCAGTGCTGTCCTGTGACA-3') was calculated by using the $2-\Delta \Delta \mathrm{Ct}$ formula [26].

\section{Isolation and Cell Culture of Primary VSMCs}

VSMCs were collected from thoracic aorta of 8 week old male SD rats by utilizing a tissue digestion approach. In brief, the rats were euthanized by using $80 \mathrm{mg} / \mathrm{kg}$ of ketamine and $10 \mathrm{mg} / \mathrm{kg}$ of xylazine given via intraperitoneal injection. In the next step, the aorta in each rat was open to meticulously remove the adventitia was a pair of forceps. The aorta was sliced open longitudinally while the endothelial tissues were carefully removed by using a scalpel. Finally, the aorta was made into $1 \mathrm{~mm}^{2}$ squares and digested with $0.25 \%$ of trypsin for 10 minutes at $37 \circ \mathrm{C}$. Then, the 
141 tissue sections were cultured at $37{ }^{\circ} \mathrm{C}$ in a DMEM containing $20 \%$ of FBS. VSMCs were later

142 purified from the cultured cells by using immunocytochemistry staining.

\section{Cell culture and transfection}

144 When the VSMCs reached logarithmic growth, they were divided into the following 5 groups: 1. 145 NC group; 2. MSU group; 3. MSU + COL group; 4. MSU + ALL group; and 5. MSU + ALL + 146 COL group. In the MSU group, the cells were incubated with MSU crystals at a $5 \mathrm{mg} / \mathrm{dL}$ 147 concentration (Sigma Aldrich, St Louis, MO) for $48 \mathrm{~h}$ MSU to induce the generation of proinflammatory cytokines. In the MSU + COL group, the cells were incubated with $20 \mathrm{ng} / \mathrm{ml}$ of colchicine after they were incubated with $5 \mathrm{mg} / \mathrm{dL}$ of MSU to induce the generation of proinflammatory cytokines. In the MSU + ALL group, the cells were incubated with $5 \mu \mathrm{mol} / \mathrm{L}$ of ALLO after they were incubated with $5 \mathrm{mg} / \mathrm{dL}$ of MSU to induce the generation of pro152 inflammatory cytokines. In the MSU + ALL + COL group, the cells were incubated with both 5 153 $\mu \mathrm{mol} / \mathrm{L}$ of ALLO and $20 \mathrm{ng} / \mathrm{ml}$ of colchicine after they were incubated with $5 \mathrm{mg} / \mathrm{dL}$ of MSU. The incubation of ALLO and colchicine lasted another $48 \mathrm{~h}$ after the $48 \mathrm{~h}$ incubation with MSU. All

155 cells were collected at the end of experiment for succeeding assays.

\section{ELISA}

157 The contents of TBARS, Catalase, TURF, GPx, MDA, IL-1b, IL-6, and TNF in collected rat 158 samples were evaluated by using commercial ELISA assay kit (R\&D Equipments, Minneapolis, $159 \mathrm{MN}$ ) in accordance to the procedure provided by the kit supplier. The reading of ELISA plates was 160 carried out by utilizing a microplate reader (ELX800, Bio-Tek, Winooski, VT).

\section{Statistical analysis}

162 Statistical analyses were done by utilizing SAS version 3.2 as well as R version 3.0.2. One-way 163 ANOVA was utilized to calculate inter group differences, which were deemed statistical 164 significant if the $\mathrm{P}$ value was less than 0.05 .

165 Results

166 Combined administration of ALL and COL suppressed the aortic vascular calcification in 167 HUC rats. 
A hyperuricemia rat model was established as described. As shown in Fig.1A, Von Kossa staining was performed to evaluate aortic vascular calcification in HUC rats treated under different conditions. Accordingly, quantitative analysis (Fig.1B) indicated that the aortic vascular calcification in HUC rats was remarkably increased, while the treatment with Allopurinol (ALL) or Colchicine (COL) alone considerably reduced the level of aortic vascular calcification in HUC rats. Furthermore, the curative effect of the combination therapy of ALL+COL was superior and the aortic vascular calcification in HUC rats was almost reduced to a level resembling that in the SHAM control. In conclusion, the combined administration of ALL and COL outperformed monotherapies of ALL and COL in terms of alleviating aortic vascular calcification in HUC rats.

\section{Combined administration of ALL and COL maintained the expression of BMP2, RUNX2, $O C$ and ALP in HUC rats.}

BMP2, RUNX2, OC and ALP are involved in the pathogenesis of HUC. Quantitative real-time PCR was performed here to analyze the expression of BMP2 (Fig.2A), RUNX2 (Fig.2B), OC (Fig.2C) and ALP (Fig.2D) mRNA in HUC rats treated under different conditions. The expression of BMP2, RUNX2, OC and ALP mRNA was dramatically elevated in HUC rats when compared with that in the SHAM control. Mono-therapy with COL or ALL could reduce the expression of BMP2, RUNX2, OC and ALP mRNA in HUC rat to a certain extent, and the combined administration of ALL and COL more dramatically abolished the up-regulation of BMP2, RUNX2, OC and ALP mRNA level to the mRNA level in the SHAM controls.

\section{Combination therapy of ALL and COL restored the dysregulation of oxidative response in} HUC rats.

In order to further explore the therapeutic effect of COL and ALL treatment on HUC rats, ELISA was carried out to evaluate the expression of several catalytic enzymes closely correlated to the oxidative stress in HUC. The expression of TBARS and MDA was notably increased in HUC rats, whereas the expression of Catalase, SOD and GPx was obviously inhibited in HUC rats. Treatment with COL or ALL alone reversed the expression of TBARS (Fig.3A), Catalase (Fig.3B), SOD (Fig.3C), GPx (Fig.3D) and MDA (Fig.3E) in HUC rats significantly, while the combined treatment with COL and ALL obstructed the up-regulation of the above-mentioned genes, which were reversed to the similar levels in SHAM controls. 

HUC rats. 221 level.

222

223

224

Inflammatory cytokines which are involved in the severity of HUC, including IL-1b (Fig.4A), IL6 (Fig.4B), TNF (Fig.4C) and TLR2 mRNA (Fig.4D) was dramatically up-regulated in HUC rats when compared with that in the SHAM control. COL and ALL mono-therapies could alleviate the expression of IL-1b, IL-6 and TNF to a certain degree. However, when COL and ALL were administrated in a combined manner, the expression of IL-1b, IL-6, TNF and TLR2 mRNA was similar to that in the SHAM control.

Combined treatment with ALL and COL maintained the expression of BMP2, RUNX2, OC and ALP in VSMCs stimulated by MSU.

A HUC cellular model was established by treating VSMCs with MSU. Further treatments with COL or ALL alone, or in a combined manner significantly up-regulated the expression of BMP2 (Fig.5A), RUNX2 (Fig.5B), OC (Fig.5C) and ALP (Fig.5D) mRNA in VSMCs. Treatment with COL or ALL alone decreased the expression of BMP2, RUNX2, OC and ALP mRNA to a certain extent, but the combined treatment with COL and ALL almost reduced the expression of BMP2, RUNX2, OC and ALP mRNA to a normal level.

Combined treatment with ALL and COL inhibited oxidative and inflammatory responses in VSMCs stimulated by MSU.

Furthermore, we evaluated the expression of oxidative enzymes and inflammatory cytokines in VSMCs treated under different conditions. MSU treatment dysregulated oxidative enzymes and inflammatory cytokines in VSMCs. Both COL and ALL treatments along showed considerable capability to maintain the levels of oxidative enzymes and inflammatory cytokines in VSMCs. Similar to the results obtained from the rat model, combined treatment of COL and ALL restored the levels of above oxidative enzymes (Fig.6) and inflammatory cytokines (Fig.7) to the normal

\section{Discussion}

When modulating signals are disturbed in gout, the phenotypic change caused by dysregulation will promote VSMCs to start abnormal differentiation into cells with various other mesenchymal 
225 functions, including adipocytes, chondrocytes as well as osteoblasts. Additionally, proof shows 226 that these abnormal phenotypic shifts promote the pathogenesis of vascular illness, including 227 atherosclerosis as well as Monckeberg's Sclerosis [27]. Without a doubt, the 228 chondrocytic/osteocytic transition of VSMCs in vascular calcification may explain why abnormal 229 differentiation could affect healthy conditions. HUC was reported to reduce the differentiation as well as expansion of osteoblasts, which might clarify the reasons for raised danger of osteopenia 231 in patients with gout [28]. Surprisingly, HUC was additionally shown to enhance the 232 differentiation of VSMCs and consequently help the progression of vascular calcification [29]. On 233 the other hand, ALL has actually been extensively used in the therapy of gout by enhancing 234 osteoblast differentiation via regulating the oxidative stress status [30]. Hydrogen peroxide was 235 revealed to influence the MAPK signaling, offering a molecular mechanism linking the 236 differentiation of VSMCs, oxidative stress, and arterial mineralization and COL has been found to 237 regulate oxidative stress [31]. In this study, we established a HUC rat model and performed Von 238 Kossa staining to analyze the severity of aortic vascular calcification in HUC rats treated under 239 different conditions. The combined administration of COL and ALL effectively inhibited aortic vascular calcification in HUC rats. As both ALL and COL has been reported to suppress the development of VC previously [30, 31] while the data of present study showed a synergistical inhibitory effect of VC in HUC animal model.

Allopurinol (ALL) is an inhibitor of xanthine oxidase and serves as an unrestricted radical scavenger along with its role in minimizing the level of uric acid in the serum. Although typically used for treating gout, allopurinol was actually been shown to alleviate high blood pressure. Some random controlled studies in populations with hyperuricemia and hypertension showed that allopurinol results in a better outcome $[32,33]$. In people with chronic cardiac failure $(\mathrm{CHF})$, a raised level of oxidative stress was actually linked to a poor prognosis [34, 35]. In addition, XO acts to play an essential pathophysiological role in cardiac arrest via the xanthineoxidase signaling $[36,37]$. Allopurinol was also shown to alleviate endothelial cell disorders, improve heart functions, minimize LV values, strength the stability of coronary micro-vasculature 99 as well as reduce death [38-41]. In this study, we performed qPCR to evaluate the expression of HUC-related genes in HUC rats and cellular models treated under differential conditions. The dysregulation of BMP2, RUNX2, OC and ALP mRNA was almost fully restored by the combined treatment of COL and ALL. It was revealed that the therapy with ALL can lower the level of blood sugar as 
well as the induction of ROS. As a result, allopurinol can reduce inflammation as well as steatosis, which is a sign of NAFLD. Lately, allopurinol is shown to subdue the activation of hemozoininduced NLRP3 inflammasomes, causing the reduction in hepatic inflammation [42]. Remarkably, it was verified that HUC could subdue the differentiation as well as the growth of osteoblasts both in vitro and in vivo. Furthermore, ALLO therapy could play a healing role in vascular calcification by minimizing the adverse impact of HUC while enhancing the differentiation as well as expansion of osteoblasts.

Colchicine (COL) acts as a type of microtubule inhibitors and is typically used to suppress or prevent inflammation in diseases such as pericarditis and gout [43]. One mechanism of action through which colchicine plays its anti-inflammatory role is by hindering the generation of NODlike receptor protein 3 (NLRP3) inflammasomes [44]. A retrospective research recommended that amongst individuals with gout, long-lasting therapy of colchicine might provide advantages in controlling the glycemic disorders $[45,46]$. Nevertheless, up to now, no RCT has actually examined the long lasting effects of colchicine on the metabolic rate of sugars in grownups with weight issues [47]. A previous research revealed that Colchicine acts as a special antiinflammatory reagent with adequate capacities to modify plaque structures [48]. Furthermore, a deficiency in VC specific inhibitors is believed to enhance the progression of VC. Insufficient activation of MatrixGLA protein, a powerful VC inhibitor requiring Vitamin $\mathrm{K}$ dependent carboxylation, might additionally add to the anti-inflammatory strength of Colchicine by inhibiting the polymerization of microtubules and the interruption of cytoskeleton [49]. Similarly, Colchicine exerts immunomodulatory effects by especially blocking the activation of NLRP3 inflammasomes while downregulating the activity of cytokines with pro-inflammatory effects [50]. Finally, colchicine can bind irreversibly to the dimers of tubulin to prevent the formation of microtubule assemblies [51]. On top of that, $\mathrm{COL}$ administration is related to The regulation of free radicals and oxidative stress status [52]. In this study, we carried out ELISA to analyze the expression of enzymes and cytokines related to oxidative and inflammatory responses. The combined treatment with COL and ALL was capable of maintaining the normal levels of enzymes and cytokines involved in oxidative and inflammatory responses in $\mathrm{HUC}$ rats and cellular models. Bothe COL and ALL are first line therapeutic agents in the treatment of GOUT and VC is an important complication of VC. The data from this study as well as previous study has identified the suppressive effect of ALL and COL on the development of VC but neither of them could 
287 completely abolish the VC. The findings of this study found that combination of ALL and COL

288 showed a synergistical therapeutic effect on the development of VC induced by HUC which shed

289 a light on the clinical application of both of the medicines to prevent the development of VC in the 290 subjects diagnosed with GOUT.

\section{Conclusion}

292 In summary, the findings of this study demonstrated that oxidative stress and inflammation were 293 involved in the pathogenesis of VC. Furthermore, both ALL and COL suppressed inflammatory 294 responses and oxidative stress. In this study, we tested the therapeutic effect of ALL combined 295 with COL in the treatment of VC in animals with hyperuricemia by studying their influence on 296 oxidative stress and inflammation.

\section{Conflict of interest}

298 None

299 Author contributions

300 YJ, MJW, LBS, BJC and QZ performed the majority of the laboratory work, statistics and data 301 analysis and participated in writing. WD, SLC and LBS were involved in writing. All authors 302 conceived and designed the experiments and drafted the manuscript.

\section{Data availability statement}

304 The data sets generated during and/or analyzed during the current study are available from the 305 corresponding author on reasonable request.

\section{$306 \quad$ Figure legends}

$307 \quad$ Fig.1

308 The increase in aortic vascular calcification in HUC rats was abolished by the combined treatment 309 with COL and ALL (\#: P value less than 0.05 compared with SHAM group; *: P value less than 3100.05 compared with HUC group; **: P value less than 0.05 compared with HUC+COL group).

311 A: Von Kossa staining of aortic vascular calcification in the rat model groups; 
B: Quantitative analysis showed that the promoted aortic vascular calcification in HUC rats was

313 abolished by the combined treatment with COL and ALL.

314 Fig. 2

315 The abnormally up-regulated BMP2, RUNX2, OC and ALP mRNA in HUC rats was restored to 316 a normal level by the combined treatment with COL and ALL (\#: P value less than 0.05 compared 317 with SHAM group; *: P value less than 0.05 compared with HUC group; **: P value less than 0.05 compared with HUC+COL group).

A: The aberrant increase of BMP2 mRNA expression in HUC rats was reduced by administration of COL or ALL, while the co-administration of COL and ALL more progressively obstructed the up-regulation in BMP2 mRNA expression.

B: The aberrant elevation of RUNX2 mRNA expression in HUC rats was reduced by administration of COL or ALL, while the co-administration of COL and ALL more progressively

324 obstructed the up-regulation in RUNX2 mRNA expression.

325

C: The aberrant elevation of OC mRNA expression in HUC rats was reduced by administration of COL or ALL, while the co-administration of COL and ALL more progressively obstructed the up-

327 regulation in OC mRNA expression.

328

D: The aberrant elevation of ALP mRNA expression in HUC rats was reduced by administration

329 of COL or ALL, while the co-administration of COL and ALL more progressively obstructed the 330 up-regulation in ALP mRNA expression.

$331 \quad$ Fig. 3

332 Combined administration with COL and ALL maintained the normal levels of enzymes involved 333 in oxidative response (\#: P value less than 0.05 compared with SHAM group; *: P value less than 3340.05 compared with HUC group; **: P value less than 0.05 compared with HUC+COL group).

335 A: Combined administration with COL and ALL reduced the elevation in TBARS expression in 336 HUC rats.

337 B: Combined administration with COL and ALL maintained the normal level of Catalase in HUC 338 rats. 
339 C: Combined administration with COL and ALL maintained the normal level of SOD in HUC rats.

D: Combined administration with COL and ALL maintained the normal level of GPx in HUC rats.

341 E: Combined administration with COL and ALL reduced the elevation in MDA expression in HUC 342 rats.

$343 \quad$ Fig.4

344 Combined administration with COL and ALL maintained the normal levels of cytokines and TLR2 345 mRNA involved in the inflammatory response in HUC rats (\#: P value less than 0.05 compared 346 with SHAM group; *: P value less than 0.05 compared with HUC group; **: P value less than 0.05 347 compared with HUC+COL group).

348 A: Combined administration with COL and ALL suppressed the level of IL-1b in HUC rats .

349 B: Combined administration with COL and ALL suppressed the level of IL-6 in HUC rats.

350 C: Combined administration with COL and ALL suppressed the level of TNF in HUC rats.

351 D: Combined administration with COL and ALL suppressed the level of TLR2 mRNA in HUC 352 rats.

$353 \quad$ Fig.5

354 The abnormal up-regulation of BMP2, RUNX2, OC and ALP mRNA in MSU-stimulated VSMCs 355 was restored by the combined treatment with COL and ALL (\#: P value less than 0.05 compared 356 with NC group; *: P value less than 0.05 compared with MSU group; **: P value less than 0.05 357 compared with MSU+COL group).

358 A: The aberrant increase of BMP2 mRNA expression in MSU-stimulated VSMCs was 359 progressively diminished by COL, ALL and COL+ALL.

360 B: The aberrant elevation of RUNX2 mRNA expression in MSU-stimulated VSMCs was 361 progressively declined by COL, ALL and COL+ALL.

362 C: The aberrant up-regulation of OC mRNA expression in MSU-stimulated VSMCs was 363 progressively decreased by COL, ALL and COL+ALL. 
364 D: The aberrant overexpression of ALP mRNA expression in MSU-stimulated VSMCs was 365 progressively reduced by COL, ALL and COL+ALL.

$366 \quad$ Fig.6

367 Combined treatment with COL and ALL maintained the normal levels of enzymes involved in 368 oxidative response in MSU-stimulated VSMCs (\#: P value less than 0.05 compared with NC group; $369 *$ : P value less than 0.05 compared with MSU group; **: P value less than 0.05 compared with 370 MSU+COL group).

371 A: Combined treatment with COL and ALL reduced the expression of TBARS in MSU-stimulated 372 VSMCs.

373 B: Combined treatment with COL and ALL maintained the normal level of Catalase in MSU374 stimulated VSMCs.

375 C: Combined treatment with COL and ALL maintained the normal level of SOD in MSU376 stimulated VSMCs.

377 D: Combined treatment with COL and ALL maintained the normal level of GPx in MSU378 stimulated VSMCs.

379 E: Combined treatment with COL and ALL reduced the level of MDA in MSU-stimulated VSMCs.

$380 \quad$ Fig.7

381 Combined treatment with COL and ALL maintained the levels of cytokines involved in 382 inflammatory responses in MSU-stimulated VSMCs.

383 A: Combined treatment with COL and ALL suppressed the level of IL-1b in MSU-stimulated 384 VSMCs.

385 B: Combined treatment with COL and ALL suppressed the level of IL-6 in MSU-stimulated 386 VSMCs.

387 C: Combined treatment with COL and ALL suppressed the level of TNF in MSU-stimulated 388 VSMCs.

\section{References}


3901 Robinson PC: Gout - An update of aetiology, genetics, co-morbidities and management. 391 Maturitas. 2018;118:67-73.

$392 \quad 2$ Richette P, Bardin T: Gout. Lancet 2010;375:318-328.

3933 Yamanaka H, Japanese Society of G, Nucleic Acid M: Japanese guideline for the 394 management of hyperuricemia and gout: second edition. Nucleosides Nucleotides Nucleic Acids 2011;30:1018-1029.

3964 Yu KH, Chen DY, Chen JH, Chen SY, Chen SM, Cheng TT, Hsieh SC, Hsieh TY, Hsu PF, Kuo CF, Kuo MC, Lam HC, Lee IT, Liang TH, Lin HY, Lin SC, Tsai WP, Tsay GJ, Wei JC, Yang CH, Tsai WC: Management of gout and hyperuricemia: Multidisciplinary consensus in Taiwan. Int J Rheum Dis 2018;21:772-787.

4005 Choi HK, Ford ES: Prevalence of the metabolic syndrome in individuals with 401 hyperuricemia. Am J Med 2007;120:442-447.

4026 Hisatome I, Kuwabara M: Hyperuricemia Plays Pivotal Role in Progression of Kidney $403 \quad$ Disease. Circ J 2016;80:1710-1711.

4047 Ryu ES, Kim MJ, Shin HS, Jang YH, Choi HS, Jo I, Johnson RJ, Kang DH: Uric acid405 induced phenotypic transition of renal tubular cells as a novel mechanism of chronic kidney disease. Am J Physiol Renal Physiol 2013;304:F471-480. Sanchez-Lozada LG, Lanaspa MA, Cristobal-Garcia M, Garcia-Arroyo F, Soto V, CruzRobles D, Nakagawa T, Yu MA, Kang DH, Johnson RJ: Uric acid-induced endothelial dysfunction is associated with mitochondrial alterations and decreased intracellular ATP concentrations. Nephron Exp Nephrol 2012;121:e71-78.

415 Nakagawa T, Mazzali M, Kang DH, Kanellis J, Watanabe S, Sanchez-Lozada LG, Rodriguez-Iturbe B, Herrera-Acosta J, Johnson RJ: Hyperuricemia causes glomerular hypertrophy in the rat. Am J Nephrol 2003;23:2-7.

42012 Ozbek E: Induction of oxidative stress in kidney. Int J Nephrol 2012;2012:465897. 
$421 \quad 13$ Nuki G: An appraisal of the 2012 American College of Rheumatology Guidelines for the 422 Management of Gout. Curr Opin Rheumatol 2014;26:152-161.

42314 Richette P, Doherty M, Pascual E, Barskova V, Becce F, Castaneda-Sanabria J, Coyfish M, Guillo S, Jansen TL, Janssens H, Liote F, Mallen C, Nuki G, Perez-Ruiz F, Pimentao J, Punzi L, Pywell T, So A, Tausche AK, Uhlig T, Zavada J, Zhang W, Tubach F, Bardin T: 2016 updated EULAR evidence-based recommendations for the management of gout. Ann Rheum Dis 2017;76:29-42.

15 Stamp LK, Chapman PT, Palmer SC: Allopurinol and kidney function: An update. Joint Bone Spine. 2016;83(1):19-24.

17 Nuki G, Simkin PA: A concise history of gout and hyperuricemia and their treatment. Arthritis

16 Alkadi H, Khubeiz MJ, Jbeily R: Colchicine: A Review on Chemical Structure and Clinical

19 Pascart T, Richette P: Colchicine in Gout: An Update. Curr Pharm Des 2018;24(6):684689. cellular redox-sensitive transcription factors in rats. Inflammation 2012;35:1549-1557. 
45225 Ben-David H, Livneh A, Lidar M, Feld O, Haj Yahia S, Grossman C, Ben-Zvi I: Toll-like receptor 2 is overexpressed in Familial Mediterranean fever patients and is inhibited by colchicine treatment. Best Pract Res Clin Rheumatol 2018;32:651-661.

26 Livak KJ, Schmittgen TD: Analysis of relative gene expression data using real-time quantitative PCR and the 2(-Delta Delta C(T)) Method. Methods 2001;25(4):402-408.

27 Yücel Ç, Omma A, Sertoğlu E, Sezer S, Turhan T, Özgürtaş T. Evaluation of atherogenic laboratory markers in Behçet's disease patients with vascular involvement. Archives of Medical Science. 2020;16(3):531-537. doi:10.5114/aoms.2018.79139.

28 Chhana A, Callon KE, Pool B, Naot D, Watson M, Gamble GD, McQueen FM, Cornish J, Dalbeth N: Monosodium urate monohydrate crystals inhibit osteoblast viability and function: implications for development of bone erosion in gout. Ann Rheum Dis 2011;70:1684-1691.

29 Wang S, Tong M, Hu S, Chen X: The Bioactive Substance Secreted by MSC Retards Mouse Aortic Vascular Smooth Muscle Cells Calcification. Biomed Res Int 2018;2018:6053567.

30 Orriss IR, Arnett TR, George J, Witham MD: Allopurinol and oxypurinol promote osteoblast differentiation and increase bone formation. Exp Cell Res 2016;342:166-174.

31 De Meyer GR, Grootaert MO, Michiels CF, Kurdi A, Schrijvers DM, Martinet W: Autophagy in vascular disease. Circ Res 2015;116:468-479.

32 Feig DI, Soletsky B, Johnson RJ: Effect of allopurinol on blood pressure of adolescents with newly diagnosed essential hypertension: a randomized trial. JAMA 2008;300:924932.

33 Soletsky B, Feig DI: Uric acid reduction rectifies prehypertension in obese adolescents. Hypertension 2012;60:1148-1156.

34 Jankowska EA, Ponikowska B, Majda J, Zymlinski R, Trzaska M, Reczuch K, BorodulinNadzieja L, Banasiak W, Ponikowski P: Hyperuricaemia predicts poor outcome in patients with mild to moderate chronic heart failure. Int J Cardiol 2007;115:151-155.

Niizeki T, Takeishi Y, Arimoto T, Okuyama H, Nozaki N, Hirono O, Tsunoda Y, Watanabe T, Nitobe J, Miyashita T, Takahashi H, Koyama Y, Kubota I: Hyperuricemia associated with high cardiac event rates in the elderly with chronic heart failure. J Cardiol 2006;47:219-228. 
36 Doehner W, Anker SD: Xanthine oxidase inhibition for chronic heart failure: is allopurinol the next therapeutic advance in heart failure? Heart 2005;91:707-709.

37 George J, Carr E, Davies J, Belch JJ, Struthers A: High-dose allopurinol improves endothelial function by profoundly reducing vascular oxidative stress and not by lowering uric acid. Circulation 2006;114:2508-2516.

38 Amado LC, Saliaris AP, Raju SV, Lehrke S, St John M, Xie J, Stewart G, Fitton T, Minhas KM, Brawn J, Hare JM: Xanthine oxidase inhibition ameliorates cardiovascular dysfunction in dogs with pacing-induced heart failure. J Mol Cell Cardiol 2005;39:531536.

39 Kogler H, Fraser H, McCune S, Altschuld R, Marban E: Disproportionate enhancement of myocardial contractility by the xanthine oxidase inhibitor oxypurinol in failing rat myocardium. Cardiovasc Res 2003;59:582-592.

40 Naumova AV, Chacko VP, Ouwerkerk R, Stull L, Marban E, Weiss RG: Xanthine oxidase inhibitors improve energetics and function after infarction in failing mouse hearts. Am J Physiol Heart Circ Physiol 2006;290:H837-843.

41 Mellin V, Isabelle M, Oudot A, Vergely-Vandriesse C, Monteil C, Di Meglio B, Henry JP, Dautreaux B, Rochette L, Thuillez C, Mulder P: Transient reduction in myocardial free oxygen radical levels is involved in the improved cardiac function and structure after longterm allopurinol treatment initiated in established chronic heart failure. Eur Heart $\mathbf{J}$ 2005;26:1544-1550.

42 Griffith JW, Sun T, McIntosh MT, Bucala R: Pure Hemozoin is inflammatory in vivo and activates the NALP3 inflammasome via release of uric acid. J Immunol 2009;183:52085220.

3 Terkeltaub RA: Colchicine update: 2008. Semin Arthritis Rheum 2009;38:411-419. Misawa T, Takahama M, Kozaki T, Lee H, Zou J, Saitoh T, Akira S: Microtubule-driven spatial arrangement of mitochondria promotes activation of the NLRP3 inflammasome. Nat Immunol 2013;14:454-460.

45 Wang L, Sawhney M, Zhao Y, Carpio GR, Fonseca V, Shi L: Association Between Colchicine and Risk of Diabetes Among the Veterans Affairs Population With Gout. Clin Ther 2015;37:1206-1215. 
51346 Klisic A, Kavaric N, Stanisic V, et al. Endocan and a novel score for dyslipidemia, oxidative 514 stress and inflammation (DOI score) are independently correlated with glycated 515 hemoglobin (HbA1c) in patients with prediabetes and type 2 diabetes. Archives of Medical $516 \quad$ Science. 2020;16(1):42-50. doi:10.5114/aoms.2019.87541.

51747 Demidowich AP, Davis AI, Dedhia N, Yanovski JA: Colchicine to decrease NLRP3518 activated inflammation and improve obesity-related metabolic dysregulation. Med $519 \quad$ Hypotheses 2016;92:67-73.

52048 Vaidya K, Arnott C, Martinez GJ, Ng B, McCormack S, Sullivan DR, Celermajer DS, Patel 521 S: Colchicine Therapy and Plaque Stabilization in Patients With Acute Coronary Syndrome: 522 A CT Coronary Angiography Study. JACC Cardiovasc Imaging 2018;11:305-316.

52349 Slobodnick A, Shah B, Pillinger MH, Krasnokutsky S: Colchicine: old and new. Am J Med $524 \quad 2015 ; 128: 461-470$.

52550 Ridker PM, Luscher TF: Anti-inflammatory therapies for cardiovascular disease. Eur Heart $526 \quad$ J 2014;35:1782-1791.

$52751 \quad$ Nakayama T, Sawada T: Involvement of microtubule integrity in memory impairment $528 \quad$ caused by colchicine. Pharmacol Biochem Behav 2002;71:119-138.

52952 Veerendra Kumar MH, Gupta YK: Intracerebroventricular administration of colchicine 530 produces cognitive impairment associated with oxidative stress in rats. Pharmacol Biochem Behav 2002;73:565-571. 

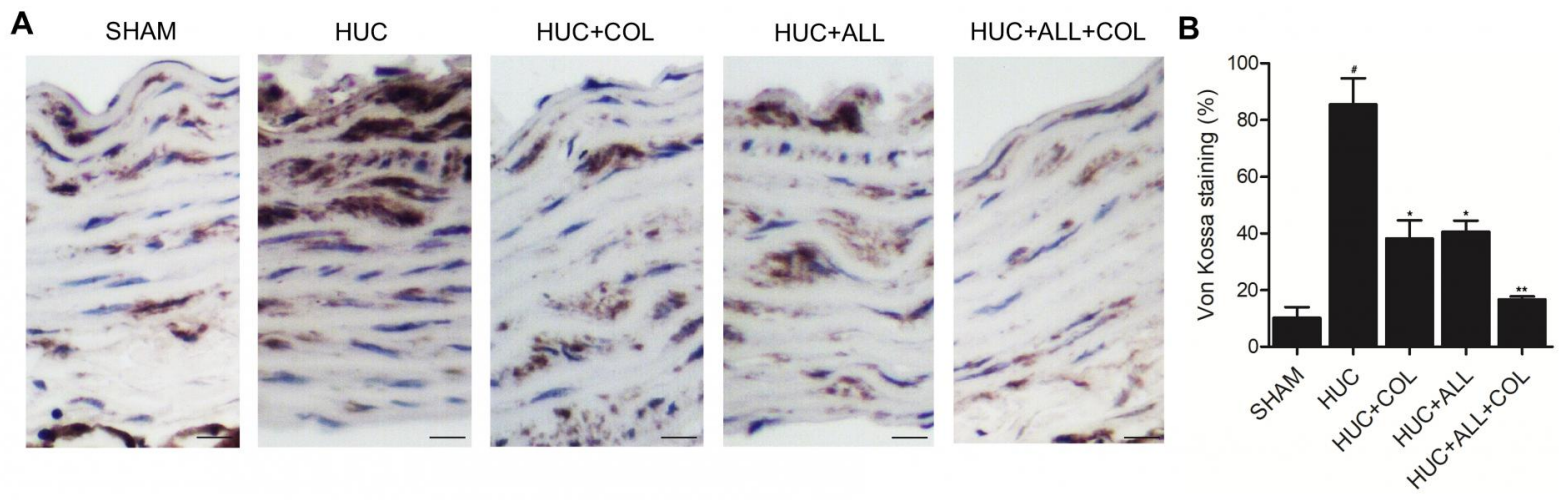

Fig.1

The increase in aortic vascular calcification in HUC rats was abolished by the combined treatment with COL and ALL (\#: P value less than 0.05 compared with SHAM group; ${ }^{*}$ : P value less than 0.05 compared with HUC group; ${ }^{* *}$ : $P$ value less than 0.05 compared with $\mathrm{HUC}+\mathrm{COL}$ group).

A: Von Kossa staining of aortic vascular calcification in the rat model groups;

$B$ : Quantitative analysis showed that the promoted aortic vascular calcification in HUC rats was abolished by the combined treatment with COL and ALL. 

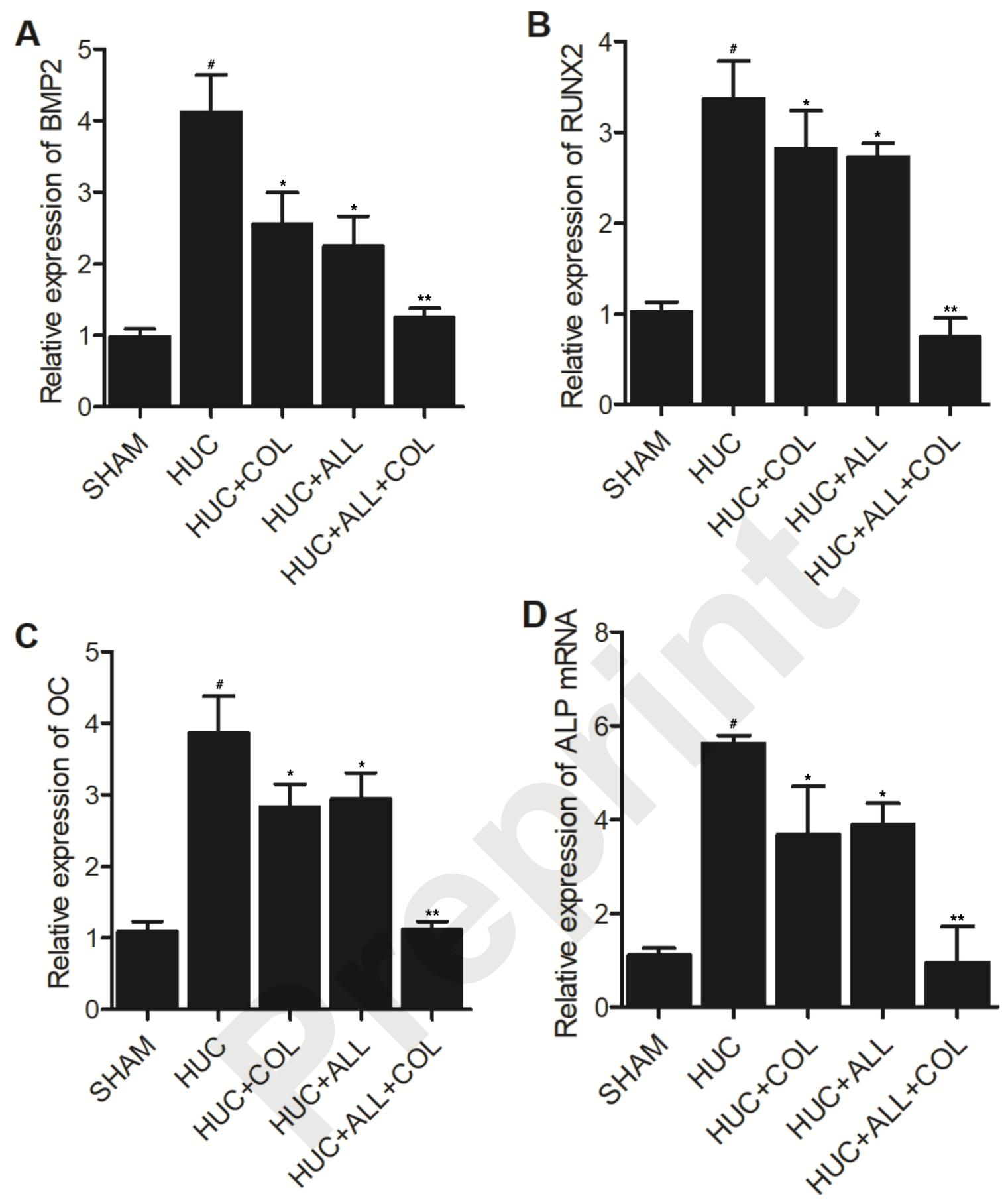

Fig.2

The abnormally up-regulated BMP2, RUNX2, OC and ALP mRNA in HUC rats was restored to a normal level by the combined treatment with COL and ALL (\#: P value less than 0.05 compared with SHAM group; *: P value less than 0.05 compared with HUC group; **: $\mathrm{P}$ value less than 0.05 compared with HUC+COL group).

A: The aberrant increase of BMP2 mRNA expression in HUC rats was progressively reduced by $\mathrm{COL}, \mathrm{ALL}$ and $\mathrm{COL}+\mathrm{ALL}$.

$B$ : The aberrant elevation of RUNX2 mRNA expression in HUC rats was progressively reduced by $\mathrm{COL}, \mathrm{ALL}$ and $\mathrm{COL}+\mathrm{ALL}$.

$\mathrm{C}$ : The aberrant up-regulation of $\mathrm{OC}$ mRNA expression in HUC rats was progressively reduced by COL, ALL and COL+ALL. 
D: The aberrant overexpression of ALP mRNA expression in HUC rats was progressively reduced by $\mathrm{COL}, \mathrm{ALL}$ and $\mathrm{COL}+\mathrm{ALL}$. 
A

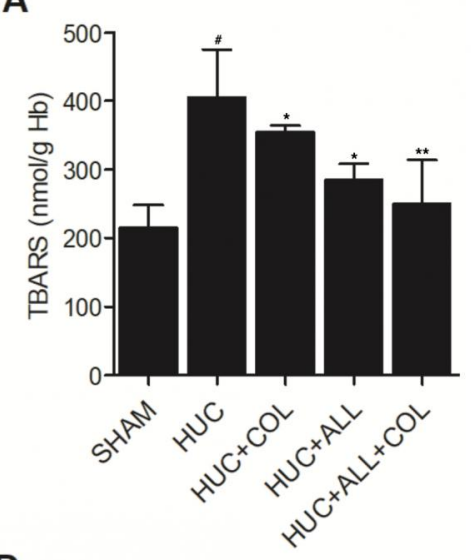

D

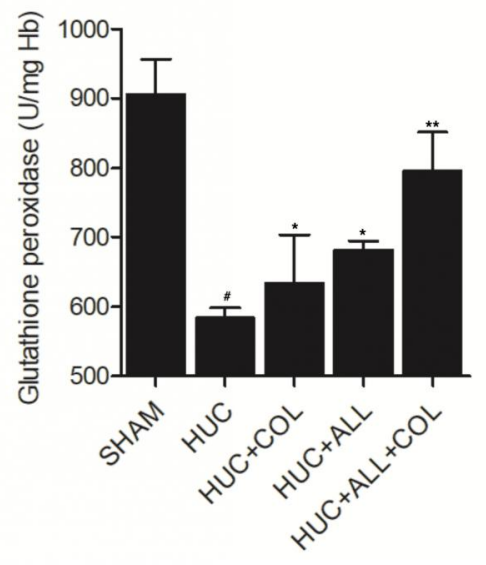

B

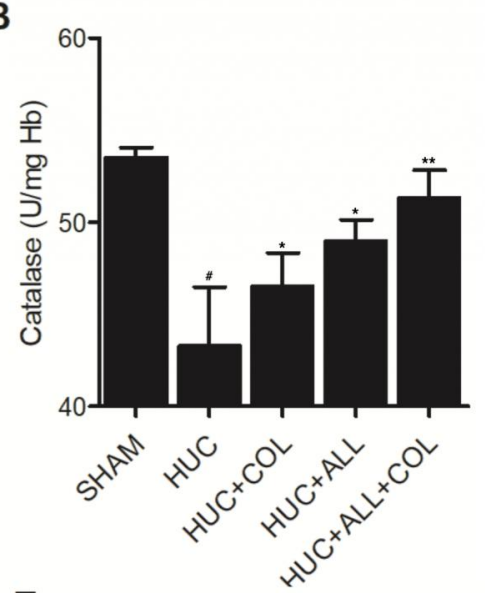

E

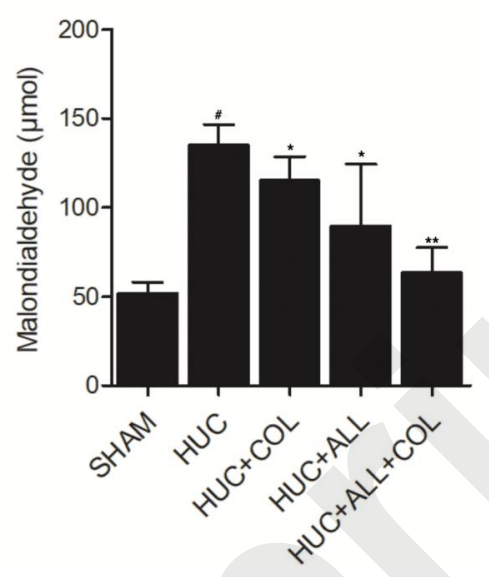

C
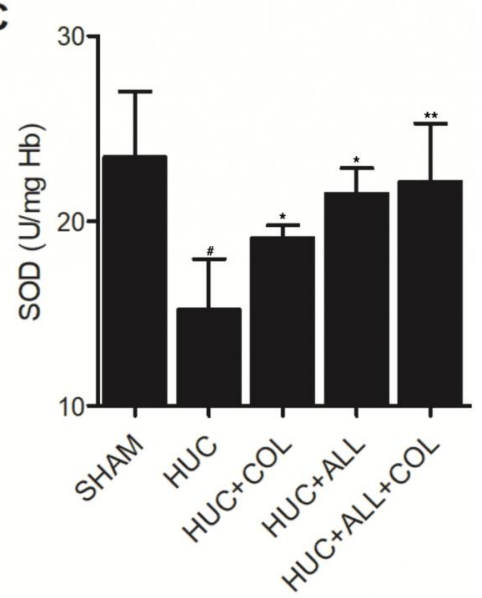

Fig. 3

Combined administration with COL and ALL maintained the normal levels of enzymes involved in oxidative response (\#: $P$ value less than 0.05 compared with SHAM group; *: $P$ value less than 0.05 compared with HUC group; ${ }^{* *}$ : $P$ value less than 0.05 compared with $\mathrm{HUC}+\mathrm{COL}$ group).

A: Combined administration with $\mathrm{COL}$ and $\mathrm{ALL}$ reduced the elevation in TBARS expression in HUC rats.

$\mathrm{B}$ : Combined administration with $\mathrm{COL}$ and $\mathrm{ALL}$ maintained the normal level of Catalase in HUC rats.

C: Combined administration with COL and ALL maintained the normal level of SOD in HUC rats.

D: Combined administration with COL and ALL maintained the normal level of GPx in HUC rats.

E: Combined administration with $\mathrm{COL}$ and $\mathrm{ALL}$ reduced the elevation in MDA expression in HUC rats. 
A

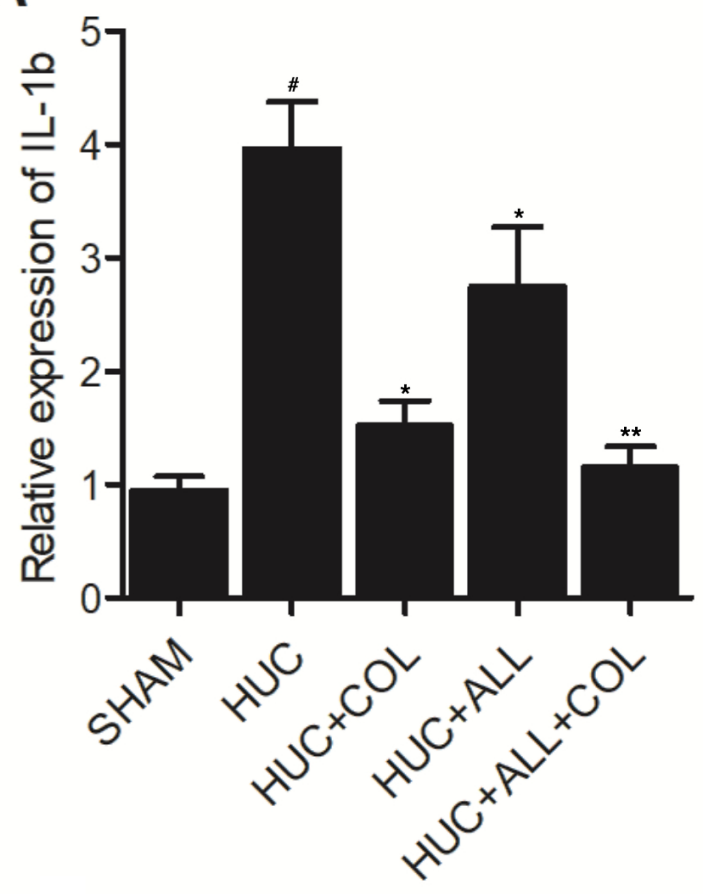

C

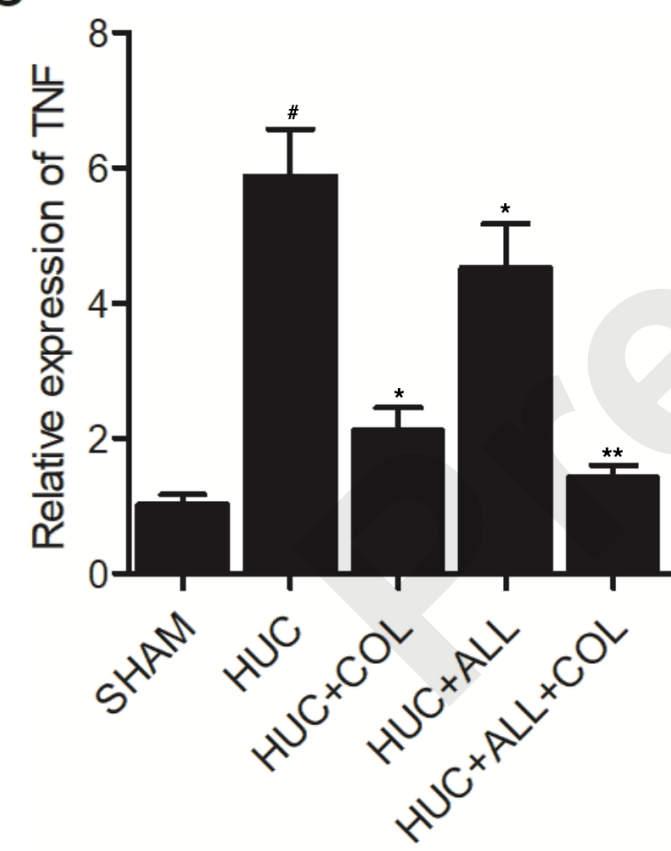

B
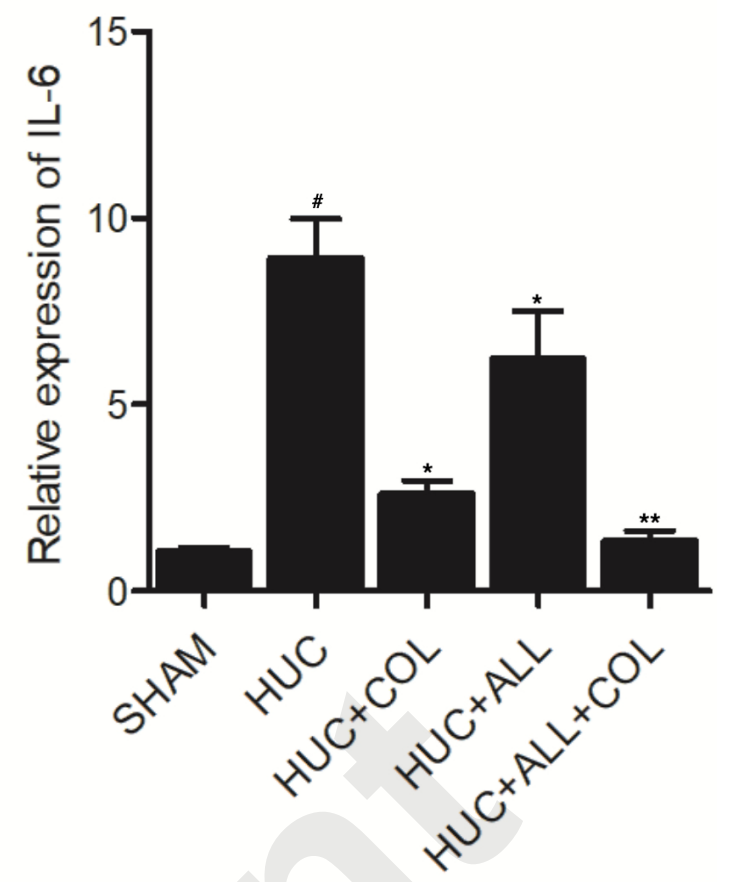

D
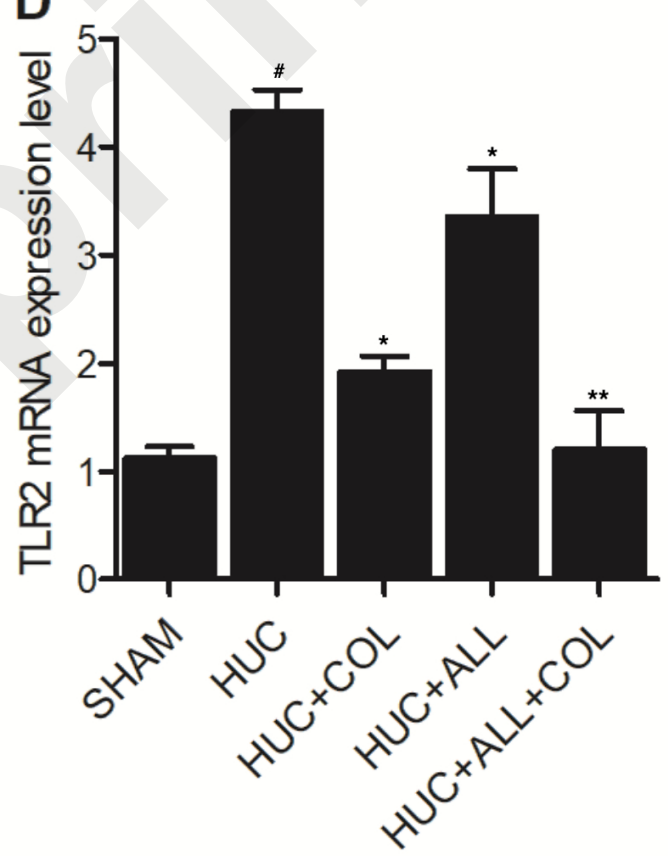

Fig.4

Combined administration with COL and ALL maintained the normal levels of cytokines and TLR2 mRNA involved in the inflammatory response in HUC rats (\#: P value less than 0.05 compared with SHAM group; *: P value less than 0.05 compared with HUC group; **: P value less than 0.05 compared with $\mathrm{HUC}+\mathrm{COL}$ group).

A: Combined administration with COL and ALL suppressed the level of IL-1b in HUC rats .

B: Combined administration with COL and ALL suppressed the level of IL-6 in HUC rats.

C: Combined administration with COL and ALL suppressed the level of TNF in HUC rats.

D: Combined administration with COL and ALL suppressed the level of TLR2 mRNA in 
HUC rats. 
A

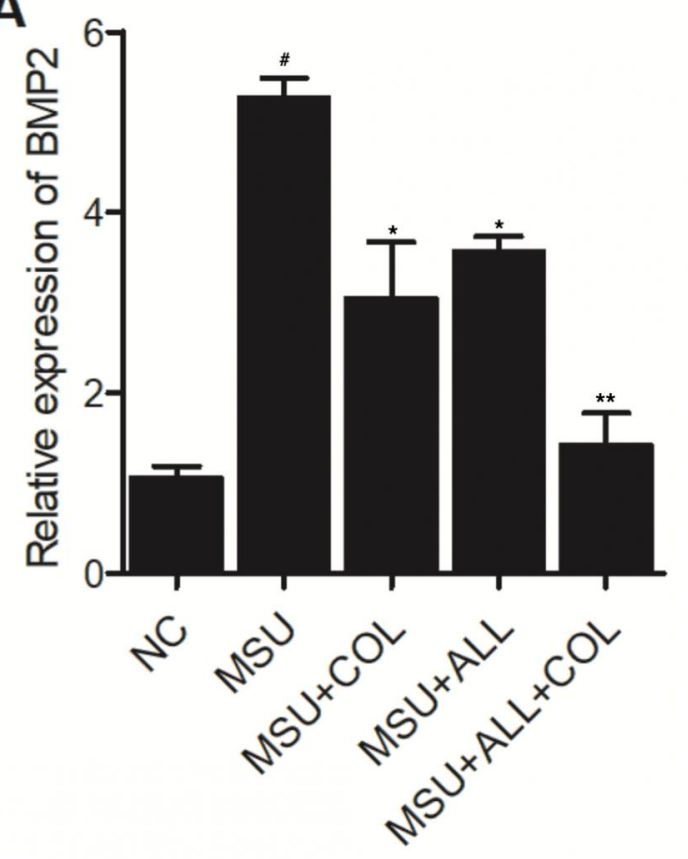

C

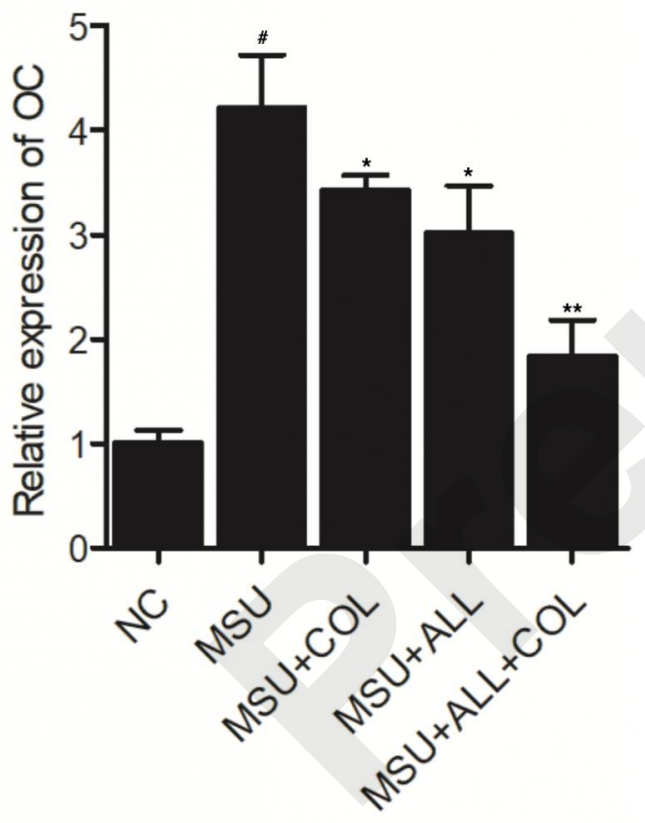

B
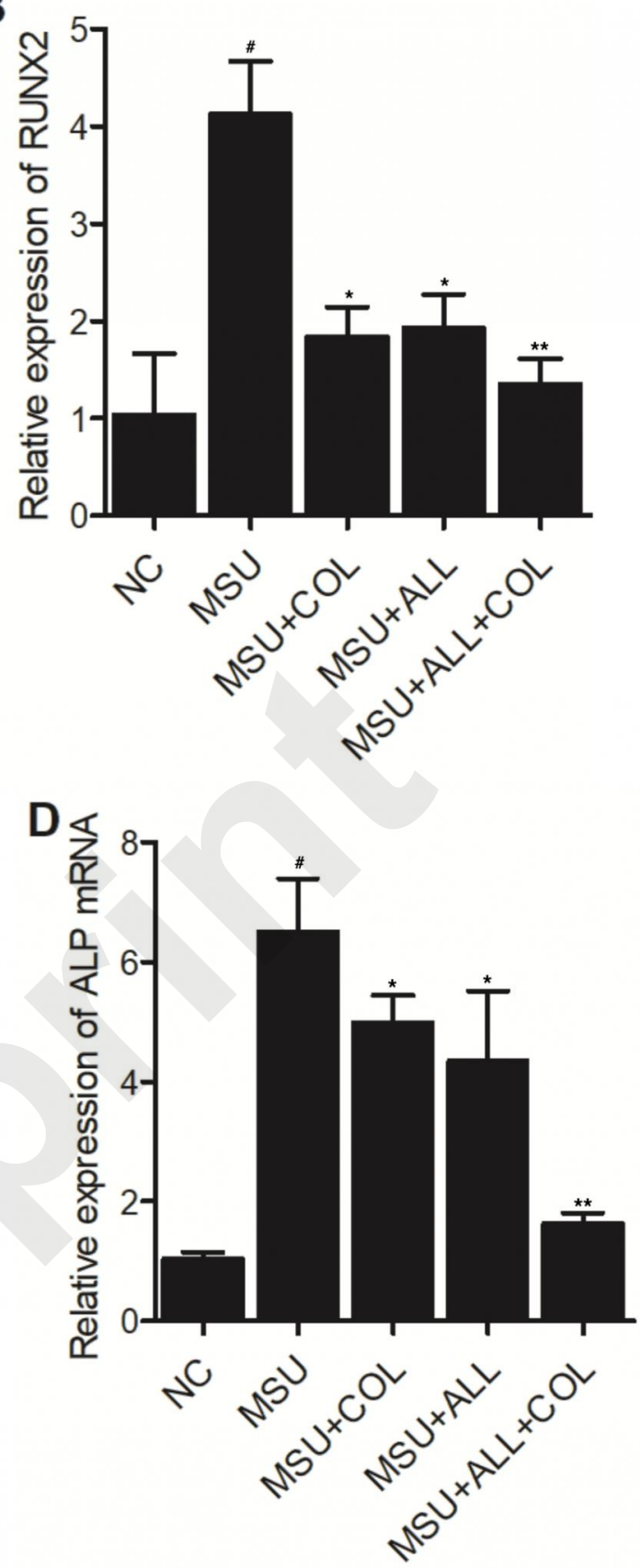

Fig.5

The abnormal up-regulation of BMP2, RUNX2, OC and ALP mRNA in MSU-stimulated VSMCs was restored by the combined treatment with COL and ALL (\#: P value less than 0.05 compared with NC group; *: P value less than 0.05 compared with MSU group; **: P value less than 0.05 compared with $\mathrm{MSU}+\mathrm{COL}$ group).

A: The aberrant increase of BMP2 mRNA expression in MSU-stimulated VSMCs was progressively diminished by COL, ALL and COL+ALL.

B: The aberrant elevation of RUNX2 mRNA expression in MSU-stimulated VSMCs was progressively declined by COL, ALL and COL+ALL.

C: The aberrant up-regulation of OC mRNA expression in MSU-stimulated VSMCs was progressively decreased by COL, ALL and COL+ALL.

D: The aberrant overexpression of ALP mRNA expression in MSU-stimulated VSMCs was 
progressively reduced by $C O L, A L L$ and $C O L+A L L$. 
A

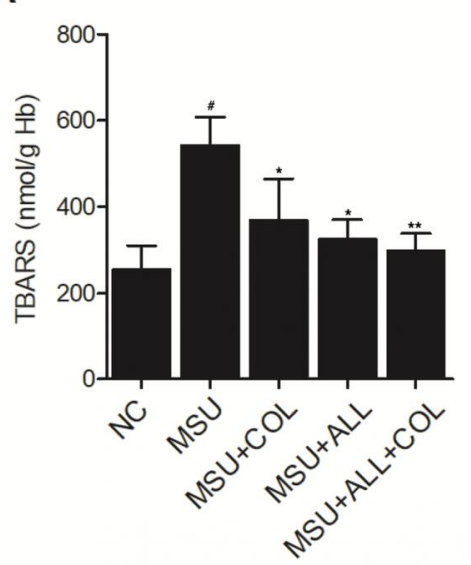

D

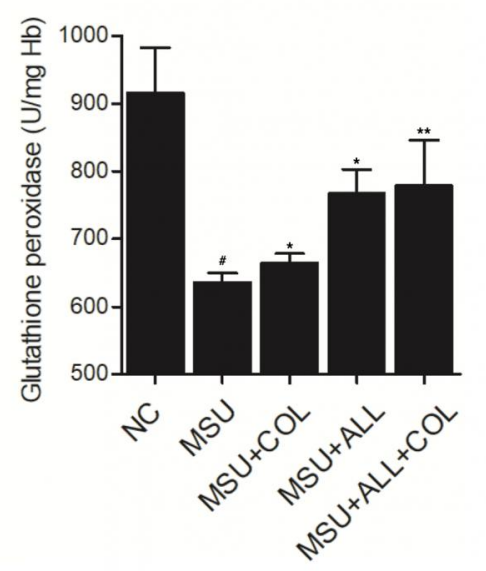

B

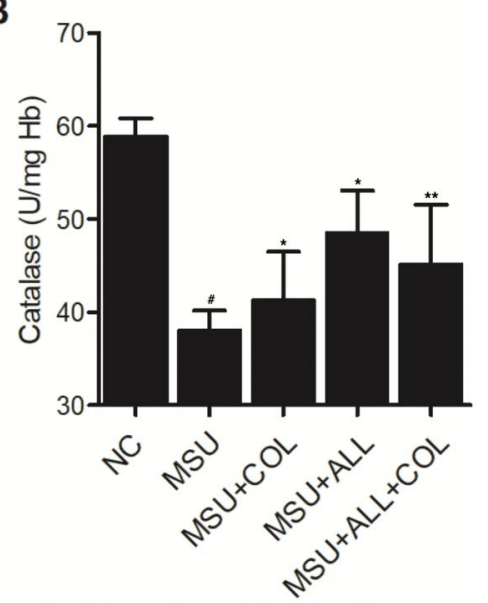

E

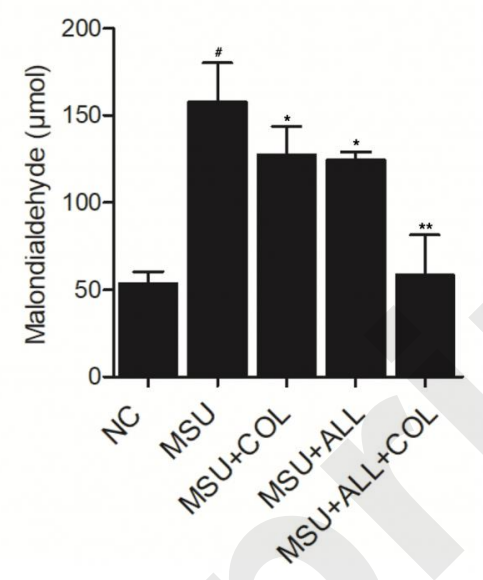

C

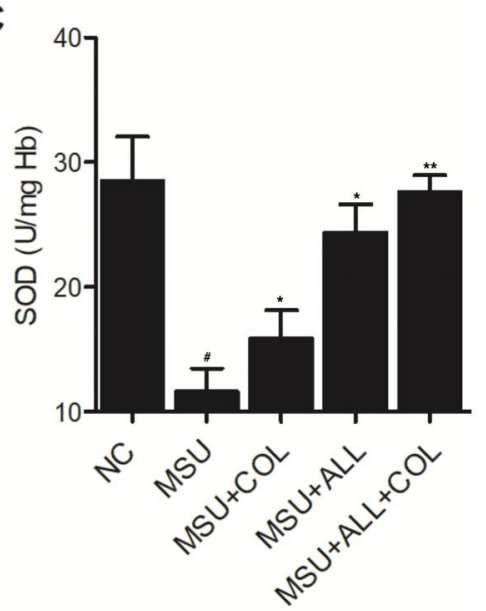

Fig.6

Combined treatment with $\mathrm{COL}$ and $\mathrm{ALL}$ maintained the normal levels of enzymes involved in oxidative response in MSU-stimulated VSMCs (\#: $P$ value less than 0.05 compared with NC group; *: P value less than 0.05 compared with MSU group; **: P value less than 0.05 compared with MSU+COL group).

A: Combined treatment with COL and ALL reduced the expression of TBARS in MSUstimulated VSMCs.

B: Combined treatment with COL and ALL maintained the normal level of Catalase in MSUstimulated VSMCs.

C: Combined treatment with COL and ALL maintained the normal level of SOD in MSUstimulated VSMCs.

D: Combined treatment with COL and ALL maintained the normal level of GPx in MSUstimulated VSMCs.

$\mathrm{E}$ : Combined treatment with COL and ALL reduced the level of MDA in MSU-stimulated VSMCs. 

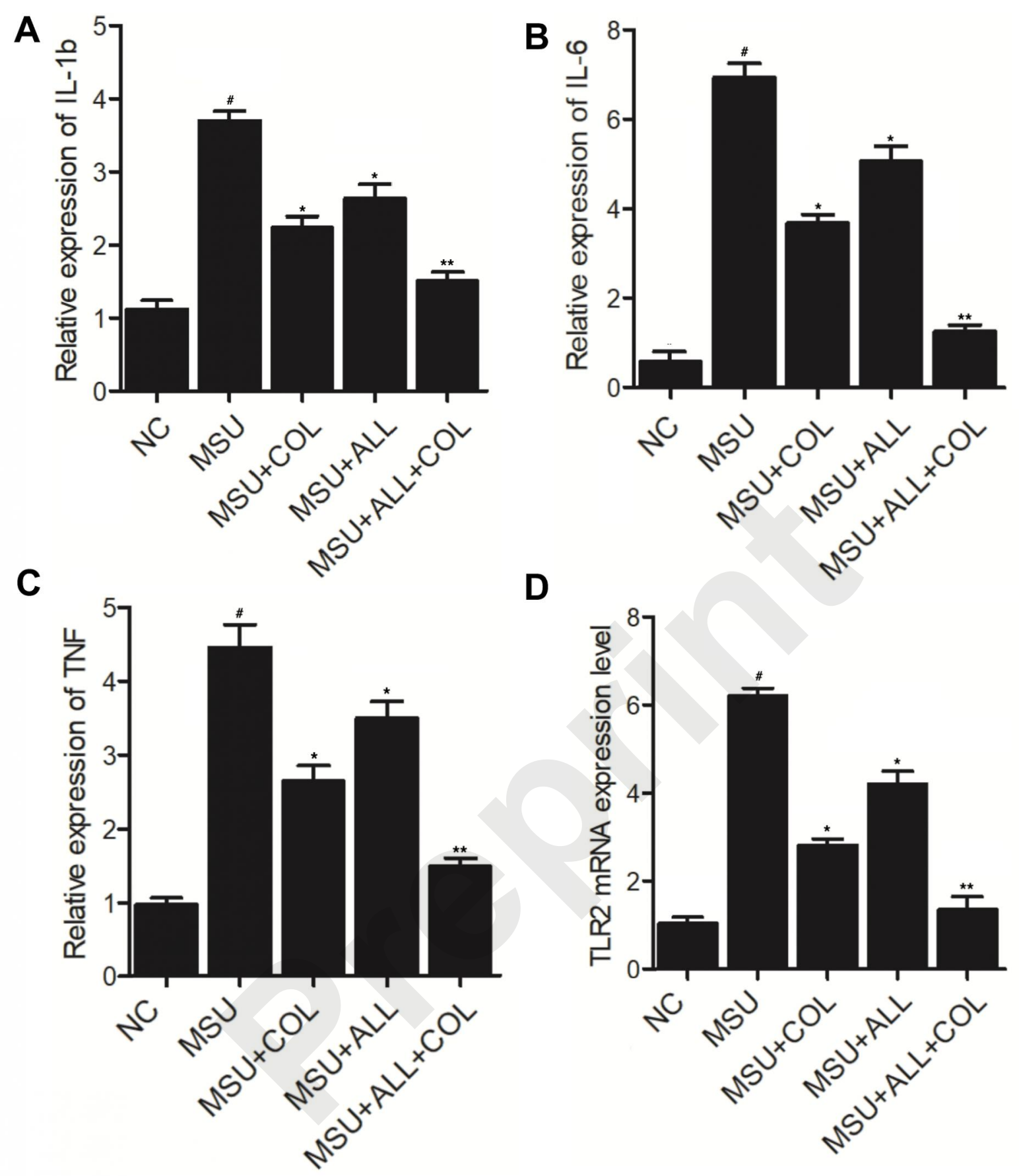

Fig.7

Combined treatment with COL and ALL maintained the levels of cytokines involved in inflammatory responses in MSU-stimulated VSMCs.

A: Combined treatment with COL and ALL suppressed the level of IL-1b in MSU-stimulated VSMCs.

B: Combined treatment with COL and ALL suppressed the level of IL-6 in MSU-stimulated VSMCs.

C: Combined treatment with COL and ALL suppressed the level of TNF in MSU-stimulated VSMCs. 